\title{
Individualized masks and respirators for COVID-19 patients and involved medical staff
}

\author{
Sarvin Sanaie ${ }^{\oplus}$, Ata Mahmoodpoor ${ }^{2 *} \oplus$, Mohammad-Salar Hosseini ${ }^{3}$
}

Dear Editor,

Since the declaration of COVID-19 as a pandemic by the World Health Organization on March 11, 2020, healthcare providers and medical staff all over the world have been increasingly facing limited availability of personal protective equipment (PPE). In the management of COVID-19 patients, the availability of PPE is crucial to protect the frontline medical staff' ${ }^{1}$.

Adherence to PPE is a crucial point regarding its efficacy. Different studies showed that the adherence of healthcare workers is not high enough due to several reasons. One of them is a gap of knowledge about the disease and transmission method, but numerous studies have suggested that education alone does not improve PPE adherence ${ }^{2}$. Thus, identifying an effective education methodology is a crucial subsequent step for improving PPE adherence. The other important factors are time pressure, heavy workload, and unsureness about the quality and the safety.

Personalized medicine could adapt the therapeutic strategies through the appropriate response and highest safety context to ensure providing the best care. Regarding prevention and PPE, masks/respirators can be an interesting topic in COVID-19. $3 \mathrm{D}$ printing translates computer-designed virtual 3D models into physical objects. 3D printing is used in the manufacturing industry, medical and pharmaceutical research, drug production, and clinical medicine and dentistry with implications for precision and personalized medicine ${ }^{3}$.

Among these, masks/respirators are of pronounced importance, since they should be appropriately worn as they fit around the nose, mouth, chin, and face. Although ensuring proper sealing seems to be difficult, it can be optimized by the personalized 3D printed masks/respirators, specially designed for each healthcare worker. These masks/respirators can be made of poly-lactic acid nanoparticles (PLA-NP), commonly used in $3 \mathrm{D}$ printers, in a short time. Furthermore, $3 \mathrm{D}$ printing plans for customizable masks/respirators can be shared publicly through social media and be accessible for everyone. Designing disposable face shields is another vital requirement that aims to help increase PPE availability through the global emergency.

COVID-19 patients are the other overlooked, yet important, part of this equilibrium. Fundamentally, non-invasive ventilation techniques (NIV) are commonly used in non-severe forms of respiratory failure. However, if the patient's condition does not improve or even deteriorates within a short period (1-2 hours), mechanical ventilation should be considered. Nevertheless, as coronavirus or any other pathogens can be spread through NIV, this ventilation mode is not recommended in these patients. Airway devices providing $6 \mathrm{~L} / \mathrm{min}$ or more of oxygen are considered as high-flow systems, and their use is discouraged if an airborne infection isolation room is unavailable ${ }^{4}$.

We believe that designing the masks that fit the person's face (personalized masks) can eliminate the leakage and make non-invasive ventilation an appropriate option for the treatment of COVID-19 patients ${ }^{5}$. The personalized masks also help to apply gentle manual breathing with minimum leakage during bag-mask ventilation for airway management without the spreading of the virus. This not only leads to more effective and safe airway management for the patients but also helps to protect the healthcare workers from virus spreading.

\section{AUTHORS' CONTRIBUTIONS}

SS: Conceptualization, Data curation, Writing - original draft. AM: Conceptualization, Data curation, Writing - original draft. MSH: Data curation, Writing - review \& editing.

\footnotetext{
${ }^{1}$ Tabriz University of Medical Sciences, Aging Research Institute, Neurosciences Research Center - Tabriz, Iran.

${ }^{2}$ Tabriz University of Medical Sciences, Faculty of Medicine, Department of Anesthesiology - Tabriz, Iran.

${ }^{3}$ Tabriz University of Medical Sciences, Student Research Committee - Tabriz, Iran.

*Corresponding author: amahmoodpoor@yahoo.com

Conflicts of interest: the authors declare there is no conflicts of interest. Funding: none.

Received on January 26, 2021. Accepted on February 01, 2021.
} 


\section{REFERENCES}

1. Chang D, Xu H, Rebaza A, Sharma L, Cruz CSD. Protecting health-care workers from subclinical coronavirus infection. Lancet Respir Medicine 2020;8(3):e13. https://doi.org/10.1016/ S2213-2600(20)30066-7

2. Morioka S, Tajima T, Sugiki Y, Hayakawa K, Ohmagari N. Adherence to personal protective equipment use among nurses in Japanese tertiary care hospitals: what determines variability? J Hosp Infect. 2020;104(3):344-9. https://doi. org/10.1016/j.jhin.2019.11.019
3. Furlow B. Medical 3-D printing. Radiol Technol. 2017;88(5):519CT37CT. PMID: 28500106

4. Yu IT, Xie ZH, Tsoi KK, Chiu YL, Lok SW, Tang XP, et al. Why did outbreaks of severe acute respiratory syndrome occur in some hospital wards but not in others? Clin Infect Dis. 2007;44(8):1017-25. https://doi.org/10.1086/512819

5. Cheung JCH, Ho LT, Cheng JV, Cham EYK, Lam KN. Staff safety during emergency airway management for COVID-19 in Hong Kong. Lancet Respir Med. 2020;8(4):e19. https://doi. org/10.1016/S2213-2600(20)30084-9 